\title{
Identidad y el patrimonio cultural. El caso de los sitios arqueológicos de la Provincia de La Rioja. Marcelo Adrián Torres*
}

\begin{abstract}
Resumen / Identidad y el patrimonio cultural. El caso de los sitios arqueológicos de la Provincia de La Rioja.

El proyecto, desde una visión interdisciplinaria y partiendo de la perspectiva de la Comunicación, el Diseño y la Antropología, aborda la presentación y comunicación del patrimonio cultural con el objetivo de promover el encuentro entre el público y los sitios arqueológicos y los lugares históricos de alta visibilidad orientados al aprovechamiento turístico sostenible de recursos arqueológicos culturales; estimular el sentido de identidad cultural en la comunidad, contribuyendo a la resignificación del pasado regional, dejando de ser visto como el pasado de los "otros" para ser el propio; y finalmente, crear las bases para el desarrollo social y turístico de la comunidad

El trabajo de investigación ha sido realizado en el marco del Programa de Becas de Posgrado del Centro de Estudios en Diseño y Comunicación de la Facultad de Diseño y Comunicación de la Universidad de Palermo.
\end{abstract}

\section{Palabras clave}

Arte ruprestre - desarrollo social - Diseño - ícono - identidad corporativa - patrimonio cultural - turismo.

\section{Summary / Identity and cultural heritage. The case of the archeological locations in La Rioja Province.}

Seen from an interdisciplinary view and from the perspective of Communication, Design and Anthropology, the project tackles with the presentation and communication of the cultural heritage with the purpose of promoting the reunion between people and the archeological locations and easy to spot historical places oriented to the sustainable tourist utilization of cultural archeological resources; encouraging the sense of cultural identity within the community, therefore going far towards a re-significance of the regional past, which stops being perceived as a past belonging to "other people" to become their own; and finally, laying the foundations for a social and tourist development of the community.

The research work presented has been done within the framew ork of the Postgraduate Scholarship Program of the Design and Communication Studies Centre of the School of Design and Communication, Universidad de Palermo,

\section{Keywords}

Cave painting - corporative identity - cultural heritage - Design - icon - social development - tourism.

\section{Resumo / Identidade e o patrimônio cultural. 0 caso dos sítios arqueológicos da Província de La Rioja.}

O projeto, desde uma visão interdisciplinar e partindo da perspectiva da Comunicação, do Design e da Antropologia, aborda a apresentação e comunicação do patrimônio cultural com o objetivo de promover o encontro entre 0 público e os sítios arqueo lógicos e os lugares históricos de alta visibilidade, orientados ao aproveitamento turístico sustentável de recursos arqueológicos culturais; estimular o sentido de identidade cultural na comunidade, contribuindo à re- significação do passado regional, deixando de ser visto como o passado dos "outros" para ser o próprio; e finalmente, criar as bases para o desenvolvimento social e turístico da comunidade.

0 trabalho de pesquisa que se apresenta têm sido realizado no marco do Programa de Becas de Pós- graduação do Centro de Estudos de Design e Comunicação da Faculdade de Design e Comunicação da Universidade de Palermo.

\section{Palavras chave}

Arte rupestre - desenvolvimento social - Design - ícone - identidade corporativa - patrimônio cultural - turismo. 
El trabajo de investigación ha sido realizado en el marco del Programa de Becas de Posgrado del Centro de Estudios en Diseño y Comunicación de la Facultad de Diseño y Comunicación de la Universidad de Palermo.

Durante mucho tiempo, cualquier visitante que se acercarse a un sitio arqueológico percibía un lugar mal conservado e incomprensible para la mayoría de ellos ya que se restringía únicamente al ámbito académico. Este desencuentro entre profesionales y público en general está cambiando lentamente al elevarse el nivel cultural, el nivel de educación y existir más tiempo libre para dedicarse al ocio, que en muchas ocasiones se traduce en una mayor demanda cultural. De alguna manera, los sitios arqueoló gicos empiezan a ser tratados como auténticos bienes de consumo y por tal motivo, su presentación al público debe prestar una importante atención.

El ICOMOS, Consejo Internacional de Monumentos y Sitios-, expresa la importancia que tiene el uso turístico de los recursos culturales y naturales, recomendando ampliar su difusión (Carta de Turismo Cultural -ICOMOS, 1976).

Esto da cuenta que el patrimonio cultural representa un gran valor artístico, histórico y cultural para la socie-dad, y por lo tanto forman parte de la personalidad e identidad de nuestro patrimonio.

Sin embargo, la mala conservación y falta de comunicación del pasado como así también la desarticulación entre el pasado y el presente por parte de la comunidad, hacen que se produzca un desencuentro entre público, comunidad y patrimonio; y por lo tanto una desvalorización del mismo.

Por tal motivo, cuando gestionamos la imagen patrimonial incorporamos bienes culturales de una manera racional y estimulamos el sentido de identidad cultural en la comunidad. La puesta en valor de sitios arqueológicos e históricos en función de su aprovechamiento turístico, remite a los pobladores del área en la que se encuentran a la consideración de su pasado reciente 0 remoto y colabora en la formación de la identidad cultural regional.

Por lo tanto, el trabajo pretende ser un aporte para la presentación y comunicación del patrimonio cultural, para hacerlas accesibles física e intelectualmente a los ciudadanos, de modo que puedan contemplar, disfrutar y comprender las obras que son herencia de la capacidad creativa de los pueblos y signos de su identidad.

\section{Características del Parque Provincial Ischigualasto} El Parque Provincial Ischigualasto (provincia de San Juan) abarca 60.369 hectáreas. Se encuentra ubicado a 83 $\mathrm{Km}$. al norte de la localidad de San Agustín de Valle Fértil y el área de amortiguación del Parque incluye las poblaciones de Baldes del Rosario y Baldecitos que suman unos 300 habitantes entre ambas. En el año 2000 , junto con el Parque Nacional Talampaya, fue declarado "Patrimonio Natural de la Humanidad" por la UNESCO.

\section{Aspectos naturales}

El denominado Parque Provincial Ischigualasto o Valle de la Luna se sitúa en el sector noreste de la provincia de San Juan, departamento de Valle Fértil, próximo al límite con la provincia de La Rioja, colindando con el Parque Nacional Talampaya.

Geográficamente se caracteriza por una vegetación desértica, principalmente arbustos, cactáceas, y árboles del desierto. El clima es desértico con poca lluvia, usualmente durante el verano, y temperaturas extremas, de $10^{\circ}$ grados en invierno a $45^{\circ}$ en el verano. La fauna presenta una particular población de guanacos de gran valor turístico por su dócil comportamiento.

El Parque constituye el único lugar en le mundo que posee las siete formaciones de sedimentos continentales del Período Triásico, con un abundante registro fósil, el cual revela la evolución de la vida de los vertebrados y la naturaleza de los paleoambientes.

Hay siete formaciones geológicas del Período Triásico. La más antigua es la Formación Talampaya, seguido por la Formación Tarjados, ambos sin fósiles de vertebrados, pero con algunas huellas y plantas. El Triasico Medio está representado por las Formaciones Chañares, Ischichuca y Los Rastros. La Formación Chañares representan la fauna antes de la aparición de los dinosaurios, y La Formación Ischigualasto representan los primeros dinosaurios.

\section{Aspectos históricos}

El resultado de los estudios realizados en el Parque ha permitido identificar dos momentos de ocupación, el primero de ellos ocurrido en el periodo prehispánico y el segundo correspondiente a tiempos históricos tardíos del siglo XIX y comienzos del siglo XX.

El segundo momento de ocupación es el que interesa en este trabajo ya que se presenta acotado a un tiempo menor a un siglo y esta relacionada al derrotero de los arrieros de vacunos a Chile. De esta ocupación se dispone información sobre la presencia de alojos, corrales, marcas de ganado grabadas en los bloques de rocas dispuestos en los lugares de transito de los arrieros. El valle de Ischigualasto constituyó una importante vía vinculada a la actividad de transporte de ganado de fines del siglo XIX y principios del XX. (Podestá, Rolandi, Ré, 2004)

\section{Aspectos socioeconómicos}

La actividad de mayor envergadura hasta la actualidad en la zona es la ganadería extensiva que se remonta a épocas coloniales en función de la demanda de ganado, bovino y equino ejercida desde Chile, Bolivia y Perú. Ha tenido épocas de esplendor en el siglo XIX hasta alrededor de 1930, asociadas al tráfico comercial con Chile. En cuanto a la actividad agrícola el departamento cuenta con una agricultura de escasa importancia y se orienta al consumo familiar y en otros casos incorpora capital y tecnología orientada al mercado.

La explotación forestal históricamente estuvo fuertemente ligada a demandas externas y orientada a soportes para parrales, postes para alambrados, cera de retamo, madera para durmientes y leña. Actualmente la explotación forestal no aparece ligada al desarrollo de una actividad económica continua en la zona.

Otra de las actividades económicas desarrollada es la minería, que actualmente presenta poco dinamismo y su aporte a la producción provincial es relativamente baja, con marcadas oscilaciones a causa de problemas 
de mercado, distancias e infraestructura vial deficitaria. La artesanía constituye una fuente alternativa de ingresos para las unidades domésticas. Son conocidas las tejedoras que realizan sus obras con recursos de la zona y en telares criollos. Los hombres se destacan en sus trabajos en cuero, lana, madera y metales para la obtención de monturas, trenzados, estribos, pellones, cuchillos que se comercializan fundamentalmente en la zona a turistas, en mercados artesanales y comercios de la capital provincial.

Respecto al turismo aproximadamente 30.000 personas visitan el parque cada año. La mayoría de los turistas vienen de Buenos Aires, seguido por Córdoba y Mendoza. Cerca de 2000 son visitantes extranjeros, principalmente de Europa, Japón y los Estados Unidos. La franja etaria de mayor incidencia en las visitas es la de 30 a 50 años de edad con un 59\%, le sigue la de 20 a 30 años con un $22 \%$ y el resto menores de 20 años y mayores de 50 años.

\section{Objetivo}

El reconocimiento previo de estos valores y su protección tienen como objetivo en este proyecto brindar un aporte para facilitar la elaboración de un diagnóstico sobre los atributos o rasgos significativos de los recursos culturales en relación con la comunidad involucrada. En este sentido, un tipo de investigación de estas características servirá como herramienta para realizar un aporte a toda estrategia de comunicación que se quiera realizar para el desarrollo del turismo cultural.

\section{Marco teórico-metodológico}

El marco teórico apunta a considerar al "patrimonio" como la base fundamental, tangible e intangible, de la identidad de una comunidad: elementos y valores a través de los cuales socialmente nos reconocemos y somos reconocidos. (M olinari et al, 2000)

Todo manejo patrimonial comprende recursos culturales. Para los propósitos de este trabajo se considera " recurso cultural" a todo vestigio del trabajo humano que constituya evidencia de la diversidad y variabilidad de las actividades y relaciones de individuos o sociedades y sus interrelaciones con el medio ambiente natural, en una perspectiva espacial y temporal. La designación de un recurso cultural para su evaluación se fundamenta en su "valor histórico" -en sentido amplio, prehistórico e histórico- derivado de su asociación a un aspecto de la historia humana y/o su evolución (APN).

La asignación de valor constituye una guía metodo-lógica que marcará las prioridades de manejo en toda actividad que afecte a los recursos culturales. Estos pueden ser: - Valor histórico, derivado de su asociación a un aspecto de la historia humana y/o su evolución.

- Valor social o simbólico, determinados recursos culturales poseen una función activa en la vida de las sociedades contemporáneas ya sea como un referente de identidad por el cual una sociedad o grupo humano se reconoce y es reconocida.

- Valor educativo, son las condiciones relativas a la riqueza e importancia disponible que presenta un recurso cultural junto con otras características como accesibilidad, visibilidad y seguridad, control.

- Valor estético, es la belleza que pueda tener un recurso cultural y/o su asociación con su entorno natural de referencia.

- Valor económico, es cuando existen razones para su aprovechamiento sustentable con fines de explotación que suponga un desarrollo para la comunidad relacionadas con el mismo.

Se considera que tanto la comunidad como los recursos culturales pertenecen a un mismo sistema, en la cual se van readaptando y modificando continuamente a través del tiempo en un mismo espacio. Entender esta adaptación implica una metodología que se fundamenta en comprender la interacción de las comunidades locales con los recursos culturales y que servirá de sustento para la elaboración de un diagnóstico sobre la imagen que tiene la comunidad respecto de los recursos culturales del área al que pertenecen.

Esta puesta en valor de los sitios arqueológicos e históricos sirve no sólo para su aprovechamiento turístico, sino también permite a la comunidad local que se encuentre con su pasado reciente o remoto colaborando en la formación de la identidad cultural regional. Esto es importante si se aspira al desarrollo del turismo cultural, ya que ellos son los que tendrán la responsabilidad de comunicar hacia fuera, por medio de su cultura la imagen que pretendan dar.

De esta forma patrimonio e identidad se asocian así con el concepto de imagen. Se define imagen como la estructura mental que se forman los habitantes 0 públicos respecto de los recursos culturales como resultado del procesamiento de toda la información relativa al mismo. Cuando se habla de la estructura mental que se forman los habitantes se refiere a los atributos que ellos le otorgan a los recursos culturales del área de influencia ya sea tangible o intangible. (Capriotti, 1999) Definimos públicos como el conjunto de personas relacionadas con los recursos culturales y naturales debido a alguna relación o vínculo particular, y por lo cual en función de esta interacción organizan sus percepciones y relaciones con el patrimonio.

Analizando el roly posición del público con respecto a los recursos culturales será posible conocer cómo lo perciben, y comprender sus intereses para actuar en consecuencia. (Capriotti, 1999)

Para observar la interrelación entre los recursos culturales y la comunidad involucrada se la analizará desde dos perspectivas: a- El inventariado de los recursos culturales. b- El análisis cultural de la comu-nidad local. a- El inventariado de los recursos culturales: Para este caso se tendrá en cuenta el manejo de los recursos culturales llevados a cabo por los científicos en el área de investigación que comprende:

- El inventariado de los recursos culturales existentes, - La generación de información científica sobre ellos. b- El análisis cultural de la comunidad: Entendemos el concepto de cultura en el sentido de ver el conjunto de normas, valores y pautas de conducta compartidas por todos en relación con su entorno. Esto es importante para la formación de la imagen de la localidad que se pretende trabajar, ya que marca las pautas de comportamiento de los miembros de la localidad.

Primero se buscará establecer cuáles son sus orientaciones y cuál es el grado de arraigo de cada una de esas 
o rientaciones. Para ello se estudiarán diferentes factores que servirán de "señales" para determinar los valores y creencias compartidos por los miembros de la comunidad. Podemos definir factores antropológicos a los comportamientos de la comunidad-creencias, normas, ritos, mitos, tabúes, jergas lingüísticas-. Una vez obtenidos los datos se los analizarán para saber la orientación de la cultura actual, permitiendo mostrar la valoración obtenida por cada una de las orientaciones que han sido seleccionadas como importantes. Posteriormente una vez definidos las dos perspectivas, se establecerá en base a las orientaciones, el análisis comparativo entre la cultura actual y las investigaciones arqueológicas para observar cuales son los atributos en los cuales la comunidad se siente identificada y en cuales no. En función del análisis comparativo de la etapa anterior surgirá una serie de posibilidades de actuación sobre la cultura de la localidad para saber el grado de integración y concientización en relación al patrimonio y qué estrategia de comunicación es posible desarrollar.

\section{Análisis}

a- El inventariado de los recursos culturales.

Los recursos culturales llevados a cabo por los investigadores en el área de investigación comprenden las siguientes características:

1. Tecnología Lítica. Alguno de los sitios en los que se realizaron recolecciones exclusivas de materiales líticos puede caracterizarse como talleres de extracción de formas-base, debido a la abundancia de núcleos y nucleiformes. Los grupos tipológicos de los artefactos formatizados son por lo general asimilables a tareas de procesamiento: Artefactos con retoques sumarios en filos laterales, raederas, puntas entre muescas, raspadores, cepillos con filos festoneados, además de fragmentos de artefactos formatizados no diferenciados. De las tres puntas de proyectil halladas, dos de ellas pertenecen al sitio Los Pasantes y pueden reconocerse como pertenecientes a momentos tardíos. Por su parte, en Agua de la Peña se recolectaron una punta lanceolada espesa de cuarzo similar a las que pertenecen los sistemas de cazadores recolectores tempranos de la región. (Gambier 2000)

2. Tecnología Cerámica: La cerámica muestra variaciones que parecen ser tanto cronológicas como de lugar de origen. Es posible establecer una asignación cronológica relativa de la cerámica hallada por su similitud con otros ejemplares hallados en el área Centro-Oeste dentro de claros contextos estratigráficos. La cerámica decorada -y creemos que también la ordinaria- pertenece a dos estilos diferentes que se sucedieron en el tiempo. El más antiguo, el denominado Calingasta en su repre-sentación incisa, fue asignado a finales del período temprano. El segundo, el Angualasto, aparece tanto en las líneas curvas y rectas pintadas en tonos rojos y negros sobre fondos ante y rojo como en líneas rectas incisas. (Gambier, 1999)

3. Grabados Rupestres. En los trabajos de campo realizados se documentaron gran cantidad de sitios con arte rupestre. Los grabados registrados en Portezuelo de las Piedras M arcadas, Agua de la Peña, Kiosco y en algunos bloques de Piedra Pintada son manifestaciones pertenecientes a las poblaciones prehispánicas del valle.
Además se destacan aquellos sitios con represen-taciones grabadas de marcas de ganado que son testigos del arreo de vacunos que se desarrolló en la región. La actividad económica predominante en la zona de Valle Fértil, donde se localiza nuestra área de estudio, es la ganadería extensiva desde épocas coloniales. (Michieli 1992) La hoyada de Ischigualasto constituyó una vía vinculada al transporte de ganado, por lo menos, desde fines del siglo XIX hasta las primeras décadas del XX, cuando esta actividad entró en franca decadencia.

En su mayoría las marcas consisten en las iniciales de los nombres y apellidos de los dueños del ganado pero también hay atributos del culto cristiano, utensilios del hogar y otros signos de difícil identificación. Se han registrado también números, nombres propios, leyendas y algunas fechas -trece en total- que permiten situar cronológicamente la ejecución de estos motivos entre 1870 y 1915. (Rolandi, Podestá et al, 2002)

4. Estructuras de Piedras. En la localidad denominada Campo de Estructuras del Río Ischigualasto se relevaron estructuras circulares, subcirculares u ovales de diferentes características. Una estructura peculiar, vestigio de las ocupaciones subactuales del valle, es un refugio 0 alojo. Se trata de una estructura pequeña y muy baja, con techo de ramas de jarillas. Los alojos, empleados para pernoctar en forma precaria, fueron utilizados por los arrieros durante su travesía. El único caso registrado se encuentra en Agua de la Peña pero los baqueanos de Ischigualasto informan sobre la existencia de otros.

b- El análisis cultural de la comunidad local.

Para establecer el conjunto de aspectos que tiene influencias en la conformación de la cultura actual de la comunidad se buscó establecer cuáles son sus orientaciones y cuál es el grado de arraigo de cada una de esas orientaciones. Para ello se estudiaron diferentes factores que servirán de " señales" para determinar los valores y creencias compartidos por los miembros de la comunidad.

1. El Desarrollo Histórico. En este caso se debería hacer tres diferenciaciones que marcarán rumbos determinantes para el uso de los recursos culturales y para la vida de la comunidad. Estos tienen que ver con un primer momento indígena, un segundo momento con el auge de la ganadería y un tercer momento con el surgimiento de Ischigualasto como valor científico.

Respecto al primer momento, Sarmiento menciona en Recuerdos de Provincia (1850) a los huarpes que habitaron los valles de Tulún, M ogna, Jachal y las llanuras de Huanacache. Cerca de Calingasta, existen más de quinientas casas de forma circular, con atrios hacia el Oriente, todas diseminadas en desorden, y figurando en su planta trompas de aquellas que nuestros campesinos tocan haciendo vibrar con el dedo una lengüeta de acero. En Zonda, en el cerro Blanco, se pueden ver perfiles de guanacos y otros animales, plantas humanas talladas en la piedra, cual si se hubiese estampado el rastro sobre arcilla blanda. Vivían aquellos pueblos de la pesca en las lagunas de Huanacache, hacia las cordilleras se alimentaban de la caza de los guanacos que pasean en manadas la gramilla de los faldeos.

En un segundo momento hacia fines del siglo XIX y principios del XX se produjo en la Argentina un auge de 
la ganadería. Los países limítrofes constituían una parte importante de la demanda de carne; en este escenario provincias como San Juan, si bien no tenían un papel crucial en la producción, si lo desempeñaban como paso de ganado hacia Chile y el Pacífico. La región de Cuyo tenía un intenso movimiento comercial en el cual los arrieros de vacunos cumplían una función vital. La actividad económica predominante en la zona de Valle Fértil, desde épocas coloniales, es la ganadería extensiva. (Michieli, 1992) El valle de Ischigualasto constituyó una importante vía vinculada a la actividad de transporte de ganado de fines del siglo XIX y principios del XX. El corredor natural que se encuentra al pie de las Barrancas Coloradas era aprovechado por los arrieros en su derrotero originado en las distintas provincias del oeste y centro de nuestro país y cuyo destino final se encontraba en el norte de Chile. En ese mismo corredor se encuentran las marcas de ganado sobre bloques de roca dejadas por los arrieros como testigos de su paso por la zona.

A continuación se detalla el relevamiento registrado por los investigadores en Ischigualasto (Rolandi, Podestá, 1990): Los grabados históricos incluyen una gran variedad de motivos representados.

\begin{tabular}{|lrr|}
\hline Tipos de motivos & $\mathrm{N}$ & $\%$ \\
Marcas de ganado & 831 & 56,07 \\
Iniciales & 198 & 13,36 \\
Números & 9 & 0,61 \\
Nombres & 4 & 0,27 \\
Fechas & 4 & 0,27 \\
Leyendas & 7 & 0,47 \\
Otros Figurativos & 24 & 1,62 \\
Abstractos & 225 & 15,18 \\
Indeterminados & 180 & 12,15 \\
Total general & 1482 & 100,00 \\
\hline
\end{tabular}

Tabla 1. Tipos de motivos de Piedra Pintada

\begin{tabular}{|lrr|}
\hline Tipos de motivos & $\mathrm{N}$ & $\%$ \\
Marcas de ganado & 12 & 52,17 \\
Iniciales & 4 & 17,39 \\
Números & 1 & 4,35 \\
Nombres & - & - \\
Fechas & - & - \\
Leyendas & - & - \\
Otros Figurativo & 1 & 4,35 \\
Abstractos & 2 & 8,70 \\
Indeterminados & 3 & 13,04 \\
Total general & 23 & 100,00 \\
\hline
\end{tabular}

Tabla 2. Tipos de motivos de Agua de las M arcas

En un tercer momento se había mencionado que los recursos culturales del área de Parque Ischigualasto está interrelacionado con el sistema humano y ecológico de la localidad de Baldecitos o/y Baldes de Rosario especialmente desde la década del '30 cuando los baqueanos acompañaban a los científicos en las prospecciones que realizaban en la zona. Posteriormente desde la década '50 esta actividad se extendió como una tarea de guía a los visitantes que llegaban al lugar para apreciar sus geoformas y bellezas escénicas. En la actualidad descendiente de aquellos primeros baqueanos forman parte del personal de guardaparques del Parque. Estas localidades están vinculadas a la actividad turística con una incipiente actividad artesanal relacionada al parque de dinosaurios y a la ganadería de bovinos.

1. Crecimiento y Crisis. Estos marcarán valores o pautas de conducta que afianzan su propia cultura. Son como señales que las encontramos en la misma historia de la comunidad cuando a principios de fines del siglo XIX se dio el auge de la ganadería junto con la conformación del personaje de los gauchos arrieros, quienes eran respetados y admirados por los demás. Las características fundamentales del gaucho arriero como poseedor de un conocimiento popular se distinguen la de ser un buen rastreador y baqueano cuyas funciones eran características importantes para llevar a cabo tal empresa. La importancia del arreo en esta provincia también se refleja en un censo realizado poco después de 1860, en el cual de los 32.640 varones, 4.235 se declararon arrieros de ganado (Levene, 1942: 257-258), es decir, el $13 \%$ de la población masculina.

El último arreo sucedió en 1940, con 3.500 vacunos, a pie, arreándolos, desde Rodeo -Iglesia- a Vallenar, en Chile cruzando la cordillera a más de 4.000 metros sobre el nivel del mar. Poco tiempo después el Gobierno Nacional puso en vigencia una ley para favorecer a los ganaderos de la pampa, y prohibió la salida del país del ganado en pie. Este fue el último arreo de San Juan a Chile y el comienzo de la declinación de nuestro perfil ganadero.

Respecto a los indígenas, Escolar señala que la figura del arriero aparece frecuentemente asimilada a la del indígena. Las categorías baqueano, arriero y rastreador, han caracterizado algo más que la posesión de un oficio, conocimiento, o la pertenencia a una determinada clase social, muchos autores dan a estos términos una connotación aborigen. (Escolar, 1996-97:19) Estos son percibidos por la población actual en la zona de Calingasta como personas con gran conocimiento del terreno, rutas y sitios de refugio cordilleranos, del clima, de las técnicas de manejo de ganado, de la carga y transporte de mulas, con habilidad como jinetes y rastreadores, capacidad de supervivencia y resistencia física y con conocimiento de los usos tradicionales de los recursos. Otro rasgo característico de la personalidad indígena es su permanente movilidad y este nomadismo se vincula a prácticas como la del arreo y las expediciones de caza del guanaco. (id 2001:156-157) Sin embargo, desde el punto de vista del actual gaucho arriero ellos no se adscriben como indígenas por tal motivo, para esta investigación, no son señales significativas.

2. Los actores claves. Las características personales de los miembros de la comunidad -sus creencias, valores, conocimiento, etc.- influyen en la conformación de la cultura. Estas aportaciones personales interactúan con las de otros individuos de la organización, llegando a imponerse dentro de la comunidad. Estos son los casos de los arrieros o baqueanos que influyeron en relación al Parque Ischigualasto, por ejemplo el caso de Martín Villafañe.

La relación de Villafañe con Ischigualasto se remonta a 
mediados de siglo cuando el paleontólogo Alfredo Romer llega a la región para investigar. Todo empezó cuando en 1870, el Presidente de la Nación de aquel entonces D. F. Sarmiento empieza con la explotación comercial de las minas con el propósito de adelantar el progreso de los ferrocarriles en el país. Con este estimulo la exploración geológica empezó a examinar los sedimentos no metalíferos. En 1927 el geólogo R. Rigal descubrió huellas fósiles debajo de los mantos de carbón. Posteriormente le escribe una carta en los años 30 al joven paleontólogo Alfred Romer, recién llegado a la universidad de Harvard, diciéndole que debían mirar estos sedimentos. Recién en 1958, Dr. Alfred Romer, ya con 64 años explora estos conjuntos y a mediados de Abril 1958 llegaron al Cerro Morado, habiendo buscado a Martin Villafañe en Balde del Rosario para acompañarles como baquiano.

Otros actores principales se produce cuando se forma un cuerpo de guardaparques siendo los primeros $\mathrm{M}$ anuel (Nolly) Sánchez y Dante Herrera. Sánchez fue enviado por la provincia a la escuela nacional de guardaparques en Neuquén para calificarse profesionalmente. Después él entrenó a los otros. Dante Herrera sigue y actualmente es el jefe de los guardaparques.

3. El entorno social. Se encuentra vinculado al incremento de la actividad turística de los últimos años, que tiene como elementos alusivos la temática inherente al parque de dinosaurios y de sus geoformas usando como materiales elementos vegetales y rocas del lugar. Son conocidas las tejedoras que realizan sus obras en telares criollos. Los hombres se destacan en sus trabajos en cuero, lana, madera y metales para la obtención de monturas, trenzados, estribos, pellones, cuchillos.

\section{Discusión}

En base a las características planteadas el área de investigación según la APN presenta:

- Valor histórico debido a su asociación a un aspecto de la historia humana y su evolución.

- Valor social por poseer una función activa en la vida de las sociedades contemporáneas ya sea como un referente de identidad por el cual una sociedad o grupo humano se reconoce y es reconocida.

- Valor educativo por la riqueza e importancia disponible que presenta.

- Valor estético por su belleza y su asociación con su entorno natural de referencia.

- Y por último, valor económico para su aprovechamiento sustentable con fines de explotación que suponga un desarrollo para la comunidad relacionadas con el mismo.

Considerando estos valores se pueden obtener ciertos datos interesantes permitiendo percibir la importancia que actualmente tiene el Parque Ischigualasto en relación a los valores naturales, a su valor científico por parte de la paleontología, al valor estético por su imponente geografía y a su valor económico debido al auge turístico. Por otro lado, se puede apreciar el arraigo que tuvo y que tiene aún hoy el éxito y la posterior declinación de la actividad ganadera en el Valle de Ischigualasto para la población, reflejado en los rasgos culturales como la artesanía de la lana, el cuero, los cuchillos, la yerra, etc., elementos típicos del gaucho de aquella época. Se observan rasgos de mayor arraigo hacia los recursos históricos -grabados rupestres: M arcas de ganado, estructuras: Alojos, arquitectura- perteneciente a la vida de los arrieros. Por el contrario, existe un desarraigo de todo lo relacionado con la cultura aborigen -tecnología lítica y cerámica-.

También se pueden obtener algunas "señales" orientativas en relación a la articulación que tiene el valor natural e histórico y el valor social o simbólico por parte de la comunidad local. Comprender esta articulación servirá de sustento para la elaboración de un diagnóstico sobre la imagen de los recursos culturales.

Entrevistas a los pobladores de la localidad de Baldes de Rosario dan cuenta de la importancia en sus vidas que tiene Ischigualasto en relación a su pasado debido, no tanto al valor paleontológico, sino por su valor simbólico. Es decir, todo lo que significó el arreo en estos lugares. Muchos de ellos fueron en su niñez e incluso sus antepasados, arrieros de la zona.

En este caso se puede observar un desajuste en cuanto a la percepción que presenta el Parque de Ischigualasto por su valor científico y económico; y la percepción con valor simbólico por parte de la comunidad local. Esta se ve más arraigada a la ganadería en relación al arriero y a las marcas de ganado dejadas en los bloques de roca del valle y a las leyendas que circulan por aquélla época de gloria para la localidad.

Por lo tanto, se observa que las políticas llevadas a cabo en el Parque no hacen alusión a esta temática que funcionaría como un valor agregado para el turismo. Por otro lado, la actuación mediante talleres participativos permitirá concientizar a la comunidad sobre los valores culturales y su importancia para la formación de la imagen de la localidad que se pretende trabajar, ya que marcarán las pautas de comportamiento de los miembros de la localidad como formas de identificación con el lugar.

\section{Conclusiones tentativas}

Una vez obtenida la información es importante concientizar e informar a la comunidad sobre la importancia que tienen estos recursos por los valores descriptos mediante talleres, exposiciones, etc. de manera participativa. Ahora bien, también es importante señalar la información que debe tener la investigación en relación con la publicidad, ya que generalmente su función fue promocionar el turismo cultural de la región enviando una señal fuerte y clara. Sin embargo, cuando se habla de publicidad turística es diferente, la elección de unas vacaciones no es impulsiva es algo sobre lo que se medita e impulsa a solicitar información complementaria estableciendo una relación con el operador que la vende. Por tal motivo, la investigación provee la información pertinente dentro de todo el eslabón o cadena orientada a hacer propuestas a un consumidor que se pregunta dónde ir.

Este conocimiento sobre los valores culturales en la región abre nuevas ideas para realizar lo que la UNESCO denomina "paisaje cultural" es decir presentar elementos cada vez más integrados donde confluye la naturaleza con lo cultural. En la práctica la realización de un "itinerario cultural de ruta" involucra la recuperación del contexto geográfico e histórico donde se 
establece un contacto e intercambio de culturas. Por ejemplo, La Ruta del Esclavo en África, busca rescatar la memoria común de los africanos. En nuestro caso sería factible realizar La Ruta del Arriero.

En síntesis, factores como la gestión y la formación, la comunicación y la investigación son de gran importancia para una estrategia que busque la cooperación e integración entre cultura y turismo. Para eso es importante identificar los valores patrimoniales en su sentido más abarcador evitando que se construya una falsa cultura mercantilista dirigida a la oferta turística.

Esto no significa que la comunidad involucrada deba volcarse al mercado y vender su propia historia e identidad; sino por el contrario, el objetivo del proyecto es observar desde la visión del otro, valores culturales que pueden ser integradas o no, dentro de los planes de manejo de los recursos culturales de la región en la cual la comunidad estaría involucrada para su beneficio socioeconómico.

\section{Bibliografía}

- APNL. Administración de Parques Nacionales. Memorias.

- Capriotti, Paul (1999) Planificación estratégica de la imagen corporativa. En Editorial Ariel Comunicación.

- Escolar, Diego (2001) Subjetividad y estatalidad: usos del pasado y pertenencias indígenas en Calingasta. En: S. Bandieri (coord.) Cruzando la Cordillera. La frontera argentino-chilena como espacio social. CEHIR. Facultad de Humanidades. Universidad Nacional del Comahue, 141-165

(1996-1997) Prácticas espacio-temporales, poder e identidad entre los baqueanos de los Andes sanjuaninos. Cuadernos del INAPL 17:17-39

- Gambier, M (2000) Prehistoria de San Juan. San Juan: Ansilta, Editora.

- ICOMOS

(1989). Resolución 89/2.

(1976). Carta de Turismo Cultural.

- Levene, R. (1942) Historia de la Nación Argentina, Tomo $X$. Buenos Aires: Imprenta de la Universidad.

- Michieli, Catalina Teresa (1992) Tráfico transcordillerano de ganado y la acción de los indígenas en el siglo XVII. Publicaciones del Instituto de Investigaciones

Arqueológicas y Museo 19: 21-47. Instituto de Investigaciones Arqueológicas y M useo (UNSJ). 2004 La fundación de villas en San Juan (siglo XVIII). Colección Tesis Doctorales. Sociedad Argentina de Antropología.

Molinari, R.; L. Ferraro, H. Paradela, A. Castaño y S. Caracotche (2000). Odisea del Manejo: Conservación del Patrimonio Arqueológico y Perspectiva Holística. En 2do. Congreso Virtual de Antropología y Arqueología. Extraído www.naya.org.ar.

- Podestá M. M., Rolandi D. S, Re A. (2004) Sitio con Arte Rupestre Agua de la Peña. (Parque provincial Ischigualasto Provincia de San Juan) En Informe preliminar. Instituto Nacional de Antropología y Pensamiento Latinoamericano

- Rolandi D, Podesta M., Guraieb G. Re A. y Vidal A. (2002) Informe sobre los Bienes Culturales del Parque Provincial Ischigualasto (Prov. San Juan, Argentina). Informe de avance. Informe y Base de Datos presentado al Director del Parque Provincial Ischigualasto. Buenos Aires, Instituto Nacional de Antropología y Pensamiento Latinoamericano. MS. 